ACR20/50/70 at Weeks 12 and 24, static Investigator Global Assessment of psoriasis (sIGA) of 0 or 1 (clear or almost clear) and at least a 2-point improvement from baseline, and PASI75 at Weeks 16 and 24 or experiencing selected clinically relevant safety events through week 24 .

Results: Analyses were conducted using data from 1694 subjects (for pharmacokinetics) and 1916 subjects (for exposure-response analyses). UPA model-estimated plasma exposures in subjects with PsA who received 15mg and 30mg QD doses were comparable to previously estimated exposures in subjects with RA. Body weight and methotrexate use had no clinically relevant effects on UPA exposures. There was a statistically significant relationship between UPA Cavg and the percentage of subjects who achieved Week 12 ACR50/70, Week 16 sIGA 0/1, and Week 24 sIGA 0/1 (Figure 1). No statistically significant exposure-response relationship was observed for Week 12 ACR20, Week 16 PASI75, or Week 24 ACR20/50/70 or PASI75, indicating that the $15 \mathrm{mg}$ QD exposures are approximately at the plateau of response for these endpoints. No statistically significant relationships were observed between upadacitinib Cavg and the percentage of subjects experiencing pneumonia, herpes zoster, hemoglobin $<8 \mathrm{~g} / \mathrm{dL}$, Grade $\geq 3$ lymphopenia, Grade $\geq 3$ neutropenia. There was a shallow but statistically significant exposure-response relationships with the occurrence of serious infections and decrease in hemoglobin from baseline ( $>2 \mathrm{~g} / \mathrm{dL}$ and $>2 \mathrm{~g} / \mathrm{dL}$ in combination with hemoglobin $<$ lower limit for normal)
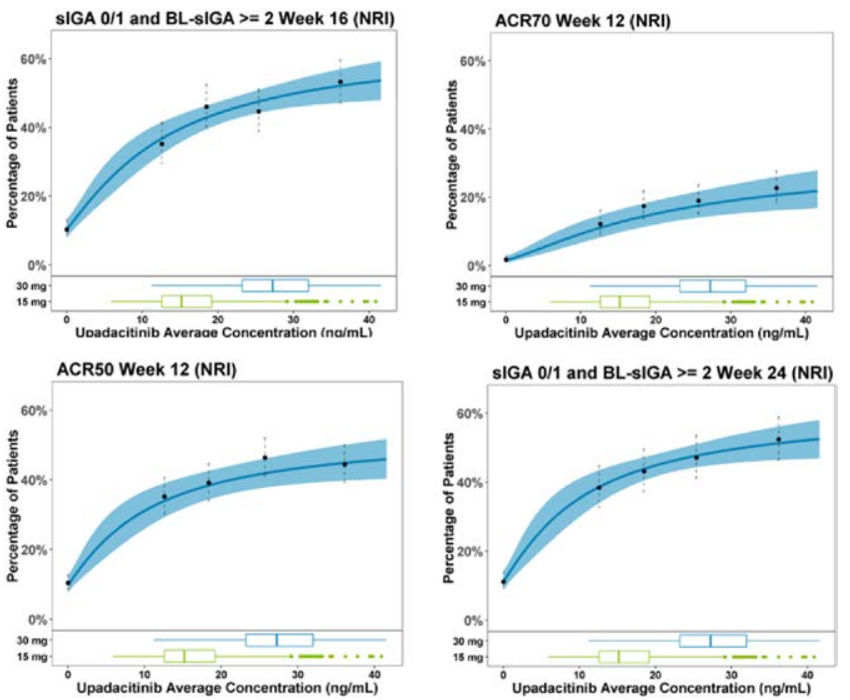

Figure 1. Observed and Model Predicted Efficacy Responses at Week 12 (for ACR50/70) or at Weeks 16 and Week 24 (for sIGA 0/1) Versus Upadacitinib Plasma Exposures

Conclusion: Exposure-response analyses demonstrated that plasma exposures associated with UPA $15 \mathrm{mg}$ QD achieves robust efficacy in subjects with PsA with limited effects on the evaluated safety endpoints. UPA plasma exposures associated with UPA 15 and $30 \mathrm{mg}$ QD are predicted to provide similar ACR responses by week 24 while a small additional efficacy benefit with UPA $30 \mathrm{mg}$ is predicted for the achievement SIGA 0/1.

REFERENCES:

[1] van Vollenhoven R, et al. Monotherapy with Upadacitinib in MTX-naïve Patients with Rheumatoid Arthritis: Results at 48 Weeks from the SELECTEARLY Study. 2019 EULAR; THU0197

[2] Fleischmann R, et al. Safety and Effectiveness of Upadacitinib or Adalimumab in Patients with Rheumatoid Arthritis: Results at 48 weeks from the SELECT-COMPARE Study. 2019 EULAR; FRI0147

Disclosure of Interests: Elena Muensterman Shareholder of: AbbVie, Employee of: AbbVie, Benjamin Engelhardt Shareholder of: AbbVie, Employee of: AbbVie, Sathej Gopalakrishnan Shareholder of: AbbVie, Employee of: AbbVie, Jaclyn Anderson Shareholder of: AbbVie, Employee of: AbbVie, Mohamed-Eslam Mohamed Shareholder of: AbbVie, Employee of: AbbVie.

DOI: 10.1136/annrheumdis-2021-eular.2182

\section{POS1055 HIGH SATISFACTION WITH USTEKINUMAB TREATMENT IN PATIENTS WITH ACTIVE PSORIATIC ARTHRITIS - FINAL RESULTS OF THE NON- INTERVENTIONAL STUDY SUSTAIN DEMONSTRATE EARLY AND LONG-TERM TREATMENT RESPONSE AND HIGH TOLERABILITY}

J. Wendler ${ }^{1}$, E. Movshovich ${ }^{2}$, N. Damann ${ }^{2}$, F. Hamann ${ }^{3}$, P. Wagener ${ }^{4}$, F. Behrens ${ }^{5}$ ${ }^{1}$ Rheumatologische Schwerpunktpraxis, Erlangen, Erlangen, Germany;

${ }^{2} J a n s s e n-C i l a g$, Neuss, Neuss, Germany; ${ }^{3}$ Gemeinschaftspraxis für internistische
Rheumatologie, Leipzig, Leipzig, Germany; ${ }^{4}$ Fachpraxis für Rheumatologie und Osteologie, Bruchhausen-Vilsen, Bruchhausen-Vilsen, Germany; ${ }^{5}$ GoetheUniversität, Rheumatologie and Fraunhofer Institut IME -TMP, Frankfurt, Germany

Background: From February 2015 to April 2020, patients with active psoriatic arthritis under treatment with ustekinumab in routine clinical care in Germany were included into the prospective, multi-center non-interventional study SUSTAIN. Objectives: Here, we present final data from SUSTAIN including final week 160 on long term efficacy and safety of ustekinumab treatment, quality of life and further patient reported outcomes.

Methods: In SUSTAIN, 337 patients were treated with ustekinumab according to routine clinical care at 75 centers for 160 weeks with documentation intervals at week 0 and 4 and then every 12 weeks. The collected data included demographics, number of swollen and tender joints, tender entheses, amount of skin symptoms (BSA and PASI), patient reported outcome concerning disease activity and pain, Health Assessment Questionnaire (HAQ), quality of life (SF-12), sleep quality (VAS), satisfaction with therapy of patient and physician, safety (adverse events [AE]/serious adverse events [SAE]), pharmacoeconomic aspects, number of patients with „Minimal Disease Activity“ (MDA) and number of patients with MDA at week 28 und 52. For this analysis, final data of all 337 patients of all documented visits were analyzed.

Results: The visit at week 4 was documented for 31 patients, at week 28 for 282 at week 52 for 216 , and at week 160 for 129 of the 337 patients. At baseline, the patients had a mean age of 53.6 years (22-85) and $192(57 \%)$ were female. The patients' mean BMI was $29.6 \mathrm{~kg} / \mathrm{m}^{2}$ (18.6-52). $54.3 \%$ of the patients had at least one TNF inhibitor as prior medication. $54.1 \%$ of the patients used MTX as concomitant medication. At time of inclusion, $73.3 \%$ of the patients showed arthritis at small and $52.2 \%$ at large joints. $16.9 \%$ showed spinal involvement and $13.1 \%$ enthesitis In the course of the treatment, the number of tender joints improved from a mean of $10.0(\mathrm{Cl} 95 \% 8.6 / 11.3)$ at baseline to $4.1(3.2 / 4.9)$ at week $28,3.6(2.7 / 4.6)$ at week 52 and $1.0(0.6 / 1.4)$ at week 160 . Number of swollen joints improved from 4.1 $(3.4 / 4.9)$ at baseline to $1.8(1.2 / 2.3)$ at week 28 , to $1.3(0.8 / 1.8)$ at week 52 and to 0.4 $(0.2 / 0.6)$ at week 160 . BSA declined from a mean of $15.6 \%$ (rage from 0 to $80.0 \%$ ) at baseline to $5.2 \%$ (0 to $60 \%$ ) at week 28 , to $3.0 \%$ (0 to $45.0 \%$ ) at week 52 and to 1.7 ( 0 to $35 \%$ ) at week 160 . PASI declined from a mean of 7.3 (range from 0 to 43.0 ) at baseline to 1.7 ( 0 to 24.8) at week 28, to 1.3 (o to 28.5) at week 52 and to 0.5 (0 to 3.2) at week 160. Efficacy of the therapy with ustekinumab was assessed as "very good" or "good" by $85.3 \%$ of the treating physicians at week 52 and by $94.1 \%$ at week 160 . The patients assessed the efficacy as "very good" or "good" by $82.8 \%$ at week 52 and by $89.8 \%$ at week 160 . At the end of the study, 96 SAEs have been documented, of which only 16 were related to ustekinumab. All in all, safety of therapy with ustekinumab was assessed as "very good" or "good" by $99 \%$ of the treating physicians after 52 weeks and by $99.2 \%$ after 160 weeks. The patients assessed the safety as "very good" or "good" by $97.5 \%$ at week 52 and by $100 \%$ at week 160 . Conclusion: The non-interventional study SUSTAIN showed relevant disease improvements and good safety with an outstanding high therapy satisfaction in patients with active psoriatic arthritis treated with ustekinumab. Treatment effect was observed as early as after 4 weeks. The treatment effects and satisfaction sustained up end of documentation at week 160 in daily practice life.

Disclosure of Interests: None declared.

DOI: 10.1136/annrheumdis-2021-eular.2495

\section{\begin{tabular}{|l|l}
\hline POS1056 COMPARATIVE EFFICACY OF TNF INHIBITORS \\
\hline
\end{tabular} VERSUS OTHER CYTOKINE INHIBITOR вDMARDS ON PSORIATIC ARTHRITIS IMPACT OF DISEASE (PSAID) SCORE AND DOMAINS}

A. Rauf ${ }^{1}$, C. Hughes ${ }^{1}$, D. Hill ${ }^{1}$, P. Kiely ${ }^{1,2} .{ }^{1}$ Rheumatology, Rheumatology, London, United Kingdom; ${ }^{2}$ St George's, University of London, Institute of Medical and Biomedical Education, London, United Kingdom

Background: Psoriatic arthritis (PsA) has many consequences, reflecting musculoskeletal and skin inflammation, with the potential to adversely affect overall quality of life. Patient reported outcome measures (PROM) assess a holistic range of aspects of quality of life, including physical and mental components, and provide broad detailed information of the impact of disease. Biologic DMARDs (bDMARDs) targeting TNF have been used to treat PsA for over 10 years whereas inhibitors of IL-17, IL-12/23 and Janus kinases (JAK) have only been available more recently. They all target differing cytokines, including JAK inhibitors which inhibit IL-12 and IL-23 signaling but not TNF signaling. Their relative impact on PROMs is unknown. Objectives: To assess, in routine care, the relative impact in PSA of TNF inhibitors (TNFi) versus non-TNFi bDMARDs, targeting IL-17, IL-12/23 and JAK, on PROMs. Methods: We performed a cross section analysis of PsA patients with established disease treated with bDMARDs and JAKi, under routine care at $\mathrm{S}$ George's University Hospital, London, UK. Patients completed the 12-item psoriatic arthritis impact of disease (PSAID) tool. The total PSAID score was calculated using the on-line EULAR toolkit (see reference). The PSAID total and individual domain scores were compared between TNFi and non-TNFi groups using the 